\title{
Null Geodesics and Strong Field Gravitational Lensing of Black Hole with Global Monopole
}

\author{
M. Sharif ${ }^{1}$ and Sehrish Iftikhar ${ }^{1,2}$ \\ ${ }^{1}$ Department of Mathematics, University of the Punjab, Quaid-e-Azam Campus, Lahore 54590, Pakistan \\ ${ }^{2}$ Department of Mathematics, Lahore College for Women University, Lahore 54000, Pakistan \\ Correspondence should be addressed to M. Sharif; msharif.math@pu.edu.pk
}

Received 16 July 2015; Revised 6 August 2015; Accepted 17 August 2015

Academic Editor: Rong-Gen Cai

Copyright (C) 2015 M. Sharif and S. Iftikhar. This is an open access article distributed under the Creative Commons Attribution License, which permits unrestricted use, distribution, and reproduction in any medium, provided the original work is properly cited. The publication of this article was funded by SCOAP ${ }^{3}$.

\begin{abstract}
We study two interesting features of a black hole with an ordinary as well as phantom global monopole. Firstly, we investigate null geodesics which imply unstable orbital motion of particles for both cases. Secondly, we evaluate deflection angle in strong field regime. We then find Einstein rings, magnifications, and observables of the relativistic images for supermassive black hole at the center of galaxy NGC4486B. We also examine time delays for different galaxies and present our results numerically. It is found that the deflection angle for ordinary/phantom global monopole is greater/smaller than that of Schwarzschild black hole. In strong field limit, the remaining properties of these black holes are quite different from the Schwarzschild black hole.
\end{abstract}

\section{Introduction}

Geodesics are associated with the motion of free particles traveling along their trajectories whose nature depends upon the spacetime. There are two types of geodesics followed by physical particles, that is, timelike and null (light-like), related to the propagation of massive and massless particles. The study of motion of massless particles such as photons is important from both astrophysical and theoretical points of view. It has been observed that light path is affected by gravity which means that path of a photon through spacetime may be bent by the gravitational field of a massive object such as a star or black hole (BH). The dynamics of test particle not only helps to understand geometrical structure of spacetime but also explains high energy phenomenon occurring near $\mathrm{BH}$ such as accretion disks where particles move in circular orbits and formation of jets in which particles escape.

Chandrasekhar [1] was the pioneer to investigate geodesic motion of a test particle around Schwarzschild, ReissnerNordström (RN), and Kerr BHs. Fernando et al. [2] constructed geodesic structure of static charged BHs of dilaton gravity and studied orbital motion of test particles. Konoplya [3] analyzed motion of both massless and massive particles around magnetized $\mathrm{BHs}$ and concluded that tidal force has considerable effect on the motion of test particles. Leiva et al. [4] studied geodesics of the Schwarzschild BH in rainbow gravity and found that geodesics remain unchanged under the influence of semiclassical effects. Guha and Bhattacharya [5] determined that the null geodesics of five-dimensional RN anti-de Sitter BH have a unique fixed point and are terminating orbits. Pradhan [6] found conditions for the existence of ISCO (inner most stable circular orbit), marginally bound circular orbit, and null circular geodesics in equatorial plane for Kerr-Newman-Taub-NUT BH.

Deflection of light in gravitational field around a massive object is referred to as gravitational lensing and an object causing deflection is called gravitational lens. Gravitational lensing is a powerful tool in cosmology as well as in astrophysics to understand distribution of mass in the large scale structures of the universe as well as cluster of galaxies and halos. It provides a useful way to estimate Hubble parameter and detection of dark mater, dark energy, exoplanet, gravitational waves, and so forth. This phenomenon is divided into two regimes: weak and strong lensing. Weak gravitational lensing produces weakly distorted images of the source. In this case, the gravitational lens is not strong enough to form 
multiple images and high magnification. It helps in the measurement of distribution of luminous as well as dark matter in the universe. If the lens is massive enough and the source and lens are highly aligned, then multiple images are formed from the background source. This phenomenon is known as strong gravitational lensing. The distortion and position of such multiple images carry important information about distribution of mass in faraway galaxies and background sources at large distance.

The theory of gravitational lensing was initially developed in weak field approximation but this approach cannot describe the phenomena like high bending of light rays and formation of infinite series of images. This motivates studying the strong gravitational lensing, which not only helps to understand these phenomena but also explains the winding of light rays multiple times around a massive object before reaching to the observer. After the pioneer work of Darwin [7], much work has been done in the context of gravitational lensing in strong field [8-11]. Virbhadra and Ellis [12] studied strong field gravitational lensing of Schwarzschild $\mathrm{BH}$ and found a sequence of relativistic images on both sides of optical axis due to large deflection of light near the photon sphere. Frittelli et al. [13] proposed an exact thin-lens equation whose accuracy was shown in the strong field. Bozza [14] developed a useful technique for spherically symmetric BHs in strong field by expanding the deflection angle near the photon sphere.

The image detection for low mass BHs is difficult but the supermassive BHs such as Sgr $A^{*}$ are an interesting example of deflection of light in strong field $[15,16]$. Ding et al. [17] considered noncommutative $\mathrm{BH}$ as gravitational lens and found effect of noncommutative parameter similar to charge by comparing with RN BH. Deng [18] studied gravitational lensing of magnetically charged $\mathrm{RN}$ BH pierced by a cosmic string in strong field and found increase in the deflection angle. Sahu et al. [19] showed that strong gravitational lensing can be used to distinguish $\mathrm{BH}$ from naked singularities. Wei et al. [20] explored strong lensing of Kerr-Taub-NUT BH and found significant effect of NUT charge. Different authors [2129] studied gravitational lensing of many other astrophysical spacetimes in strong field limit.

The fact that the universe is in the phase of accelerating expansion is a major turning point in cosmology which indicates the existence of dark energy supported by several observational evidences. Dark energy is an elusive force having large negative pressure. To understand its exact nature, several dynamical models have been proposed out of which phantom field is a strange kind of dark energy with equation of state parameter $\omega<-1$ violating the null energy condition. Exact $\mathrm{BH}$ solutions including phantom fields are called phantom BHs. Babichev et al. [30] investigated that phantom energy accretion onto a $\mathrm{BH}$ causes a continuous decrease in $\mathrm{BH}$ mass. Bronnikov and Fabris [31] found an interesting regular phantom $\mathrm{BH}$ solution which is asymptotically flat, de Sitter, and anti-de Sitter. Eiroa and Sendra [32] studied regular phantom BHs as gravitational lens and compared their results with Schwarzschild and vacuum Brans-Dicke BHs. Some people [33-35] have discussed light paths and gravitational lensing of phantom BHs.
Global monopoles are topological defects of vacuum manifold that arise from the phase transition in the early universe. Their formation depends upon the gauge symmetry breaking with a choice of suitable scalar field. It can be shown that their energy is concentrated near the monopole core into a small region. Barriola and Vilenkin [36] found static spherically symmetric BH with a global monopole. Many authors [37-42] studied physical properties of BHs with global monopole.

In this paper, we study null geodesics as well as strong gravitational lensing of spherically symmetric BHs (with ordinary and phantom global monopoles). The format of the paper is as follows. In the next section, we introduce the metric having both ordinary and global monopoles and study the behavior of null geodesics. In Section 3, we evaluate exact deflection angle using Bozza's method. Section 4 explores Einstein rings, magnifications, and observables of the relativistic images. In Section 5, we numerically study the observables for the central supermassive BH. Section 6 is devoted to the study of time delays between the relativistic images in different galaxies. In the last section, we summarize the results.

\section{Null Geodesics}

We consider static spherically symmetric $\mathrm{BH}$ with a global monopole [43]. This was obtained by global SO(3) symmetry breaking of a triplet scalar field in the Schwarzschild BH background. This is topologically different from Schwarzschild $\mathrm{BH}$ due to the existence of global monopole. The metric of this $\mathrm{BH}$ is described as

$$
d s^{2}=-f(r) d t^{2}+g(r) d r^{2}+h(r)\left(d \theta^{2}+\sin ^{2} \theta d \phi^{2}\right),
$$

where

$$
\begin{aligned}
& f(r)=g(r)^{-1}=\left(1-8 \pi \xi \eta^{2}-\frac{2 M}{r}\right), \\
& h(r)=r^{2}
\end{aligned}
$$

$M$ is the mass of $\mathrm{BH}, \eta$ is the energy scale of symmetry breaking, and $\xi$ is the term describing kinetic energy of the $\mathrm{BH}$. If $\xi=1$, it represents an ordinary global monopole originating from positive kinetic energy of scalar field [36]. If $\xi=-1$, the phantom global monopole is formed originating from negative kinetic energy of scalar field. The corresponding event horizon is

$$
r_{h}=\frac{2 M}{1-8 \pi \xi \eta^{2}} .
$$

Notice that the Schwarzschild radius is recovered for $\eta=0$, it does not have any horizon for $M=0$, and it generates a naked singularity at $r=0$. There are several phantom $\mathrm{BHs}$ having negative kinetic energy and pressure; it would be interesting to study the behavior of energy density and pressure of the $\mathrm{BH}$ with phantom global monopole. It can be seen in Figure 1 that energy density and pressure admit the properties of a phantom model which is almost similar to [35]. The expressions of $\rho_{p}$ and $P_{p}$ are given in the Appendix. 


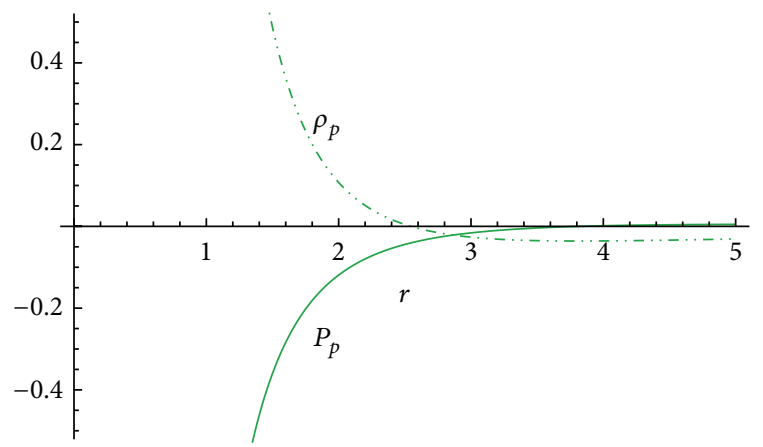

FIGURE 1: Energy density and pressure for the $\mathrm{BH}$ with phantom global monopole along $r$ for $\eta=0.15$ and $M=1$.

The Lagrangian in the equatorial plane $(\theta=\pi / 2)$ [1] for a photon traveling around a $\mathrm{BH}$ with global monopole is

$$
\begin{aligned}
2 \mathscr{L}= & -\left(1-8 \pi \xi \eta^{2}-\frac{2 M}{r}\right) \dot{t}^{2}+\frac{\dot{r}^{2}}{\left(1-8 \pi \xi \eta^{2}-2 M / r\right)} \\
& +r^{2} \dot{\phi}^{2} .
\end{aligned}
$$

Using the Euler-Lagrange equations for null geodesics and an affine parameter $q$, we have

$$
\begin{aligned}
& \dot{t}=\frac{d t}{d q}=\frac{E}{\left(1-8 \pi \xi \eta^{2}-2 M / r\right)}, \\
& \dot{\phi}=\frac{d \phi}{d q}=\frac{L}{r^{2}},
\end{aligned}
$$

where $E$ and $L$ are the energy and angular momenta per unit mass. The Hamiltonian is given as

$$
2 \mathscr{H}=-E \dot{t}+L \dot{\phi}+\frac{\dot{r}^{2}}{\left(1-8 \pi \xi \eta^{2}-2 M / r\right)}=\delta,
$$

where $\delta$ is an integral of motion and $\delta=-1,0,1$ correspond to spacelike, null, and timelike geodesics, respectively. For null geodesics, the radial equation of motion is

$$
\dot{r}^{2}+V_{\mathrm{eff}}(r)=E^{2},
$$

where $V_{\text {eff }}=\left(L / r^{2}\right)\left(1-8 \pi \xi \eta^{2}-2 M / r\right)$. Figure 2 represents the behavior of effective potential for different values angular momentum $L$. We observe that, for $\xi=-1, V_{\text {eff }}$ has only maximum values and hence only unstable circular orbits exist. For $\xi=1$, maximum value exists at $\eta \approx 0.002$ which corresponds to unstable circular orbits; however, $V_{\text {eff }}$ continuously decreases after this point leading to stable circular orbits.

\section{Deflection Angle}

Here, we study gravitational lensing in strong field limit and the effect of $\eta$ with ordinary and phantom global monopoles. We obtain deflection angle for (1) in the strong field using Bozza's technique. We can write (1) in terms of Schwarzschild

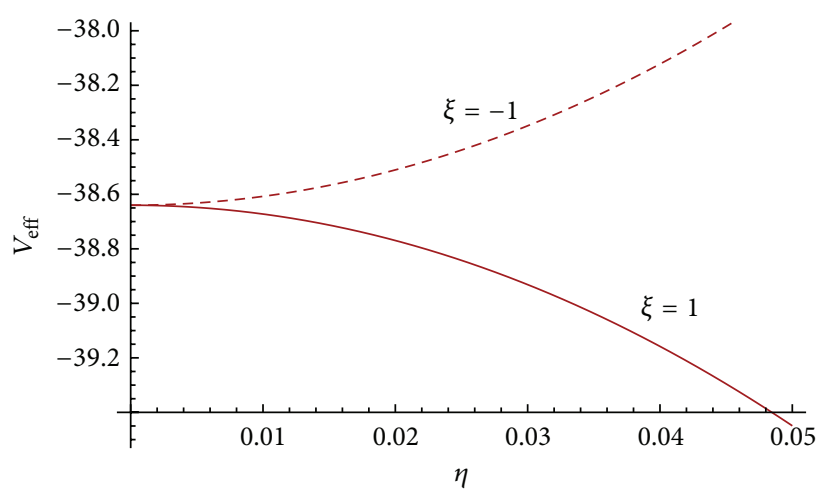

(a)

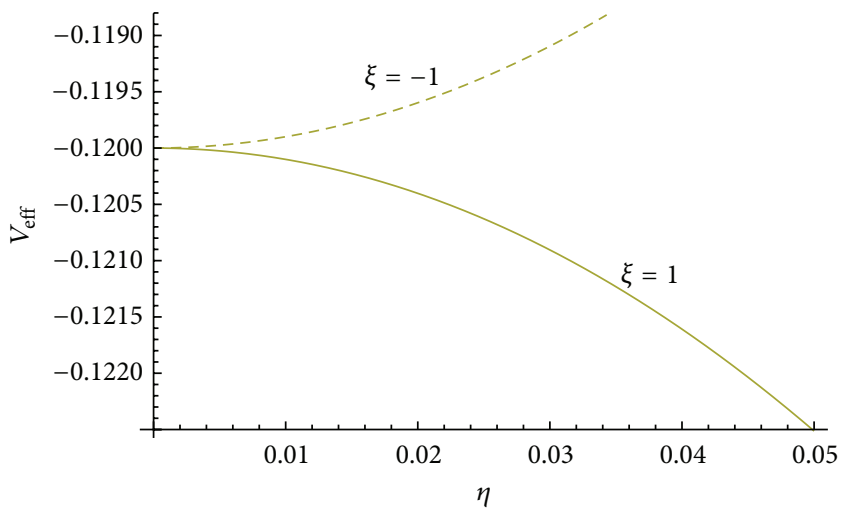

(b)

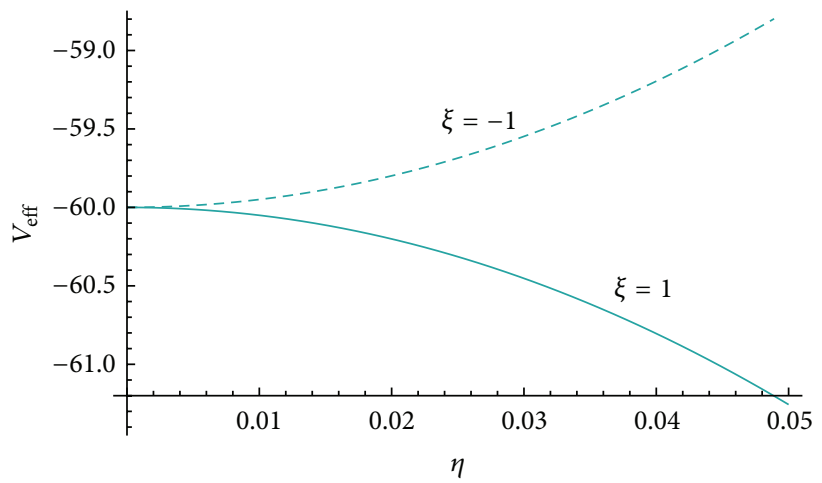

(c)

FIGURE 2: Behavior of the effective potential $\left(V_{\text {eff }}\right)$ for ordinary and phantom global monopoles as function of $\eta$ corresponding to $L=$ 3.22 (a), $L=1$ (b), and $L=5$ (c) with $M=1$ and $r=0.05$.

radius by defining the transformations $x=r / 2 M$ and $T=$ $t / 2 M$ as

$$
\begin{aligned}
d s^{2}= & -f(x) d T^{2}+g(x) d x^{2} \\
& +h(x)\left(d \theta^{2}+\sin ^{2} \theta d \phi^{2}\right),
\end{aligned}
$$

where

$$
\begin{aligned}
& f(x)=g(x)^{-1}=\left(1-8 \pi \xi \eta^{2}-\frac{1}{x}\right), \\
& h(x)=x^{2} .
\end{aligned}
$$




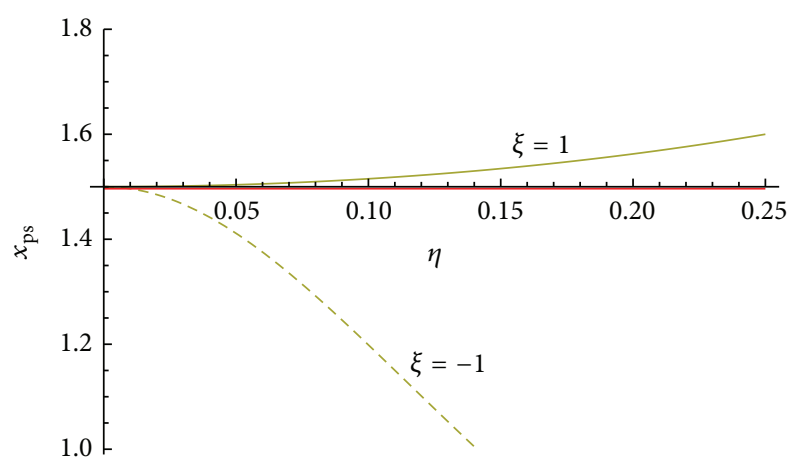

FIGURE 3: Radius of photon sphere $\left(x_{\mathrm{ps}}\right)$ versus $\eta$. Solid and dashed green curves correspond to the case of ordinary and phantom global monopoles whereas the solid red line indicates Schwarzschild limit.

The radius of photon sphere $\left(x_{\mathrm{ps}}\right)$ is the largest positive root of the equation [44]:

$$
\frac{f^{\prime}(x)}{f(x)}=\frac{h^{\prime}(x)}{h(x)}
$$

yielding

$$
x_{\mathrm{ps}}=\frac{3}{2\left(1-8 \pi \xi \eta^{2}\right)} .
$$

Figure 3 describes the behavior of $x_{\mathrm{ps}}$ for ordinary and phantom global monopoles. In the first case, the radius of photon sphere is an increasing function of $\eta$ and greater than the Schwarzschild limit while, for the second case, it shows a decreasing behavior as $\eta$ increases and is less than the Schwarzschild limit. The deflection angle for a photon (from infinity) is calculated as a function of distance of the closest approach $x_{0}[45,46]$ :

$$
\alpha\left(x_{0}\right)=I\left(x_{0}\right)-\pi
$$

where

$$
I\left(x_{0}\right)=\int_{x_{0}}^{\infty} \frac{2 \sqrt{g(x)} d x}{\sqrt{h(x)} \sqrt{f\left(x_{0}\right) h(x) / f(x) h\left(x_{0}\right)-1}} .
$$

The deflection angle depends upon the relation between $x_{0}$ and $x_{\mathrm{ps}}$ which grows if $x_{0}$ approaches $x_{\mathrm{ps}}$. Following [14], we introduce a new variable $z$ :

$$
z=\frac{f(x)-f\left(x_{0}\right)}{1-f\left(x_{0}\right)}
$$

Replacing $z$ in (13), it follows that

$$
I\left(x_{0}\right)=\int_{0}^{1} \mathscr{R}\left(z, x_{0}\right) k\left(z, x_{0}\right) d z,
$$

where

$$
\begin{aligned}
\mathscr{R} & \left(z, x_{0}\right)=2 \frac{\sqrt{f(x) g(x)}}{f^{\prime}(x) h(x)}\left(1-f\left(x_{0}\right)\right) \sqrt{h\left(x_{0}\right)} \\
& =2\left(1+8 \pi \xi \eta^{2} x_{0}\right), \\
k & \left(z, x_{0}\right) \\
& =\frac{1}{\sqrt{f\left(x_{0}\right)-\left[\left(1-f\left(x_{0}\right)\right) z+f\left(x_{0}\right)\right] h\left(x_{0}\right) / h(x)}} \\
& =\left[z \left\{\left(2-\frac{3}{x_{0}}\right)+2\left(8 \pi \xi \eta^{2}\right)\left(1-8 \pi \xi \eta^{2}\right) x_{0}\right.\right. \\
& \left.-5\left(8 \pi \xi \eta^{2}\right)\right\}+z^{2}\left\{\frac{3}{x_{0}}-1\right. \\
& -\left(8 \pi \xi \eta^{2}\right)\left(2+5\left(8 \pi \xi \eta^{2}\right)\right) x_{0} \\
& \left.+\left(8 \pi \xi \eta^{2}\right)\left(1+8 \pi \xi \eta^{2}\right) x_{0}^{2}+7\left(8 \pi \xi \eta^{2}\right)\right\}-z^{3}\left\{\frac{1}{x_{0}}\right. \\
& \left.\left.+3\left(8 \pi \xi \eta^{2}\right)^{2}+\left(8 \pi \xi \eta^{2}\right) x_{0}^{3}+3\left(8 \pi \xi \eta^{2}\right)\right\}\right]^{-1 / 2},
\end{aligned}
$$

and all functions other than the subscript 0 are evaluated at

$$
x=\left[1-f\left(x_{0}\right) z+f\left(x_{0}\right)\right] f(x)^{-1} .
$$

The function $R\left(z, x_{0}\right)$ is regular for all values of $z$ and $x_{0}$ while $k\left(z, x_{0}\right)$ diverges when $z$ tends to zero. We expand the function inside the square root (by applying Taylor's series approximation) up to second order in $z$ [14]:

$$
k_{0}\left(z, x_{0}\right)=\frac{1}{\sqrt{\zeta\left(x_{0}\right) z+\gamma\left(x_{0}\right) z^{2}}}
$$

where

$$
\begin{aligned}
& \zeta\left(x_{0}\right)=\frac{1-f\left(x_{0}\right)}{f^{\prime}\left(x_{0}\right) h\left(x_{0}\right)}\left[f\left(x_{0}\right) h^{\prime}\left(x_{0}\right)-f^{\prime}\left(x_{0}\right)\right. \\
& \left.\cdot h\left(x_{0}\right)\right]=\frac{1+8 \pi \xi \eta^{2} x_{0}}{x_{0}}\left[2 x_{0}\left(1-8 \pi \xi \eta^{2}\right)-3\right], \\
& \gamma\left(x_{0}\right)=\frac{\left[1-f\left(x_{0}\right)\right]^{2}}{2\left(f^{\prime}\left(x_{0}\right)\right)^{3} h^{2}\left(x_{0}\right)}\left[2 \left\{\left(f^{\prime}\left(x_{0}\right)\right)^{2} h\left(x_{0}\right)\right.\right. \\
& \cdot h^{\prime}\left(x_{0}\right)-f\left(x_{0}\right) f^{\prime \prime}\left(x_{0}\right) h\left(x_{0}\right) h^{\prime}\left(x_{0}\right)+f\left(x_{0}\right) \\
& \left.\left.\cdot f^{\prime}\left(x_{0}\right)\left(h\left(x_{0}\right) h^{\prime \prime}\left(x_{0}\right)-2\left(h^{\prime}\left(x_{0}\right)\right)^{2}\right)\right\}\right]=(1 \\
& \left.+8 \pi \xi \eta^{2} x_{0}\right)^{2}\left[\frac{3}{x_{0}}-1+8 \pi \xi \eta^{2}\right] .
\end{aligned}
$$


Using the above expressions, integral (13) can be separated as

$$
I\left(x_{0}\right)=I_{D}\left(x_{0}\right)+I_{\mathscr{R}}\left(x_{0}\right),
$$

where

$$
\begin{aligned}
& I_{D}\left(x_{0}\right)=\int_{0}^{1} \mathscr{R}\left(0, x_{\mathrm{ps}}\right) k_{0}\left(z, x_{0}\right) d z, \\
& I_{\mathscr{R}}\left(x_{0}\right) \\
& =\int_{0}^{1}\left\{\mathscr{R}\left(z, x_{0}\right) k\left(z, x_{0}\right)-\mathscr{R}\left(0, x_{\mathrm{ps}}\right) k_{0}\left(z, x_{0}\right)\right\} d z .
\end{aligned}
$$

When $\zeta$ is nonzero $\left(x_{0} \neq x_{\mathrm{ps}}\right)$, the order of divergence in $k_{0}$ is approximately $1 / \sqrt{z}$, while, for $\zeta$ to be zero $\left(x_{0}=x_{\mathrm{ps}}\right)$, the divergence is $1 / z$. Thus, $I_{D}$ includes divergence whereas $I_{\mathscr{R}}$ is regular as the divergent term is subtracted out [14]. By the conservation of angular momentum, the impact parameter $u$ can be defined as a function of $x_{0}[46,47]$ :

$$
u=\sqrt{\frac{h\left(x_{0}\right)}{f\left(x_{0}\right)}} .
$$

The deflection angle for the photons propagating near the photon sphere is given as

$$
\alpha(u)=-a_{1} \ln \left(\frac{u}{u_{\mathrm{ps}}}-1\right)+a_{2}+O\left(u-u_{\mathrm{ps}}\right),
$$

where $u_{\mathrm{ps}}=\sqrt{x_{\mathrm{ps}}^{3} /\left(x_{\mathrm{ps}}\left(1-8 \pi \xi \eta^{2}\right)-1\right)}$ is the value of impact parameter at $x_{0}=x_{\mathrm{ps}}$ and

$$
\begin{aligned}
a_{1} & =\frac{\mathscr{R}\left(0, x_{\mathrm{ps}}\right)}{2 \sqrt{\gamma\left(x_{\mathrm{ps}}\right)}} \\
& =\frac{1+8 \pi \xi \eta^{2} x_{\mathrm{ps}}}{\sqrt{\left(1+8 \pi \xi \eta^{2} x_{\mathrm{ps}}\right)^{2}\left[3 / x_{\mathrm{ps}}+\left(1-8 \pi \xi \eta^{2}\right)\right]}}, \\
a_{2} & =-\pi+a_{\mathscr{R}}+a_{1} \ln \sigma,
\end{aligned}
$$

Since $a_{\mathscr{R}}$ cannot be calculated directly, using Taylor's series approximation we expand it up to second order in terms of $\eta$ as

$$
a_{\mathscr{R}}=a_{\mathscr{R}, 0}+a_{\mathscr{R}, 2} \eta^{2}+O\left(\eta^{4}\right)
$$

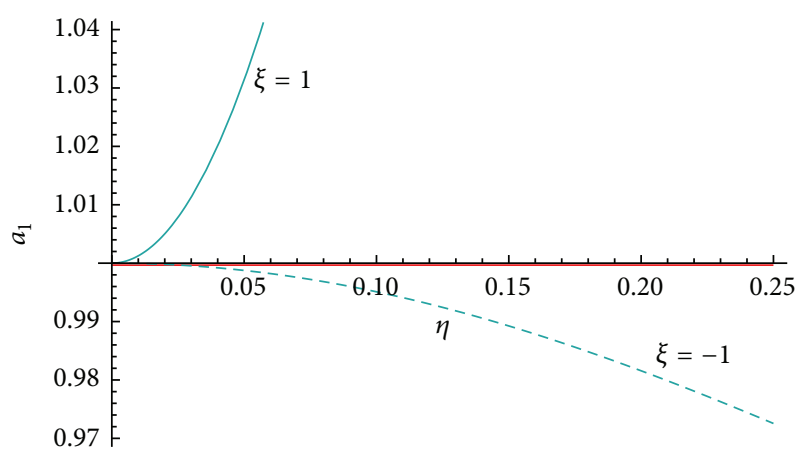

(a)

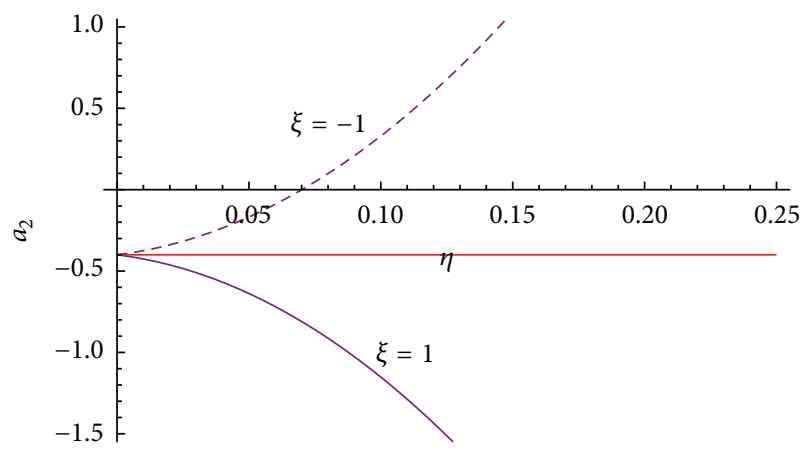

(b)

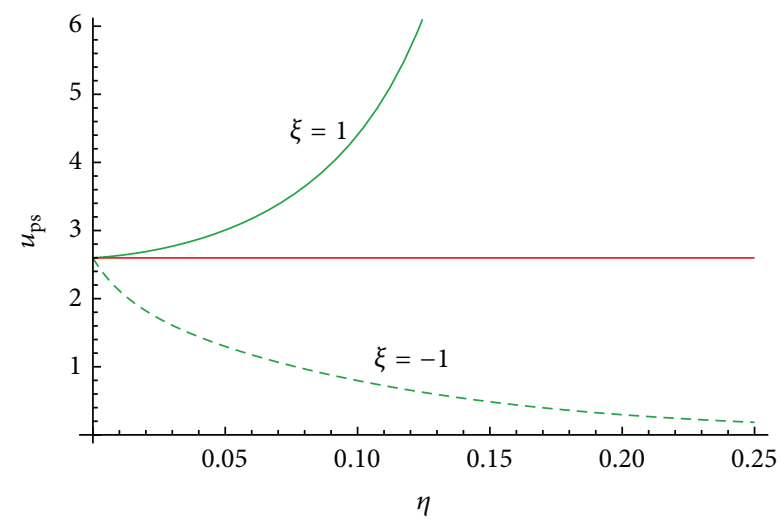

(c)

FIGURE 4: Behavior of strong field deflection coefficients $\left(a_{1}(\mathrm{a})\right.$ and $a_{2}$ (b)) and parameter $u_{\mathrm{ps}}$ (c) as functions of $\eta$ are represented by blue, purple, and green while red line shows Schwarzschild limit.

where $a_{\mathscr{R}, 0}$ corresponds to the uncharged (Schwarzschild) $\mathrm{BH}$ and $a_{\mathscr{R}, 2} \approx-2.26271(8 \pi \xi)$. The graphical analysis of the deflection coefficients $a_{1}$ and $a_{2}$ and impact parameter $u_{\mathrm{ps}}$ is shown in Figure 4 (ordinary as well as phantom global monopoles). We observe that, for $\xi=1, a_{1}$ and $u_{\mathrm{ps}}$ are increasing functions of $\eta$ and greater than the Schwarzschild limit, while $a_{2}$ diverges for large values of $\eta$ and is less than Schwarzschild limit. The situation is totally different for the phantom global monopole $(\xi=-1)$. We see that $a_{1}$ and $u_{\text {ps }}$ decrease with the increase of $\eta$ and are smaller than the Schwarzschild limit, whereas $a_{2}$ has an increasing behavior for all values of $\eta$ and is larger than the Schwarzschild limit. 


\section{Magnifications, Einstein Rings, and Observables}

In this section, we study the effect of global monopole (ordinary as well as phantom) on magnifications, Einstein rings, and observables for the relativistic images in the strong field limit. These quantities are directly related to the deflection angle by the lens equation and have been discussed in detail $[14,32]$. We briefly discuss basic equations and the results are given numerically. In the context of gravitational lensing, the deflection angle is associated with angles $\theta$ and $\beta$ describing the source and the image position. The lens equation for lens 1 situated between source $s$ and observer o is given as [12]

$$
\tan \beta=\tan \theta-\frac{D_{\mathrm{ls}}}{D_{\mathrm{os}}}\{\tan (\alpha-\theta)+\tan \theta\}
$$

The distances between observer-source, observer-lens, and lens-source are denoted by $D_{\mathrm{os}}, D_{\mathrm{ol}}$, and $D_{\mathrm{ls}}$, respectively. These distances are measured from the optical axis (line joining observer o and lens l) and are considered much greater than the horizon. We consider the case when objects are highly aligned; that is, $\beta$ and $\theta$ are small. The deflection angle for a photon traveling near the photon sphere is $\alpha=$ $\Delta \alpha_{n}+2 n \pi, n \in N$, where $0<\Delta \alpha_{n} \ll 1$. In this approximation, the lens equation takes the form [48]

$$
\beta=\theta-\frac{D_{\mathrm{ls}}}{D_{\text {os }}} \Delta \alpha_{n} .
$$

This equation describes only images on the same side of the source by taking positive $\beta$. To obtain images on the opposite side, the same equation can be solved with the source placed in $-\beta$. The deflection angle can be expressed in terms of angular position of the image $\theta$ and the observer-lens distance $D_{\mathrm{ol}}$. According to lens geometry $\left(u=D_{\mathrm{ol}} \sin \theta \approx D_{\mathrm{ol}} \theta\right)$, we have

$$
\alpha(\theta)=-a_{1} \ln \left(\frac{D_{\mathrm{ol}} \theta}{u_{\mathrm{ps}}}-1\right)+a_{2} .
$$

The behavior of deflection angle for both cases (ordinary and phantom global monopoles) can be seen in Figure 5. For the first case, $\alpha$ is a monotonically increasing function of $\eta$ while it decreases in the second case. Also, for ordinary global monopole, $\alpha$ is larger than Schwarzschild limit (after $\eta=0.1$ ), while, for phantom global monopole, the deflection angle is very much less than Schwarzschild limit as well as for ordinary global monopole. We find that a light ray passing near a $\mathrm{BH}$ with global monopole makes a large deflection angle as compared to the light ray traveling near a $\mathrm{BH}$ with phantom global monopole.

An infinite sequence of Einstein rings is given as

$$
\theta_{n}^{E}=\left(1-\frac{\varsigma_{n} D_{\mathrm{os}}}{D_{\mathrm{ls}}}\right) \theta_{n}^{0},
$$

where

$$
\begin{aligned}
\varsigma_{n} & =\frac{u_{\mathrm{ps}}}{a_{1} D_{\mathrm{ol}}} e^{\left(a_{2}-2 n \pi\right) / a_{1}}, \\
\theta_{n}^{0} & =\frac{u_{\mathrm{ps}}}{D_{\mathrm{ol}}}\left[1+e^{\left(a_{2}-2 n \pi\right) / a_{1}}\right] .
\end{aligned}
$$

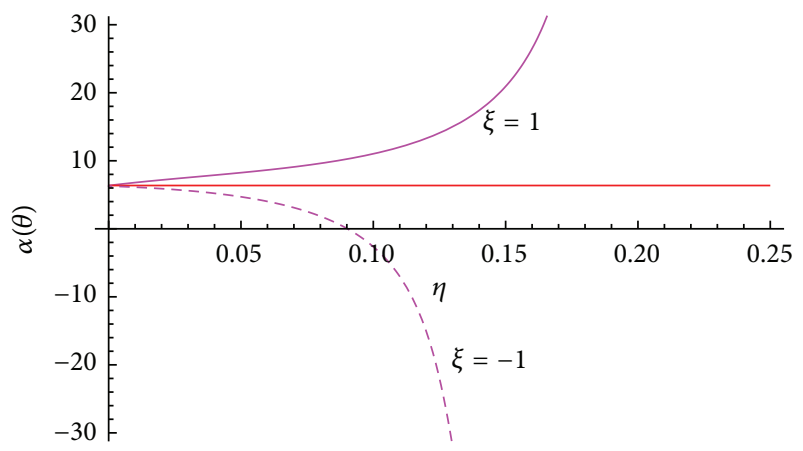

FIgURE 5: Deflection angle $\alpha$ for ordinary and phantom global monopoles evaluated at $u=u_{\mathrm{ps}}+0.003$ as a function of $\eta$, whereas the red line indicates Schwarzschild limit.

The magnification of the $n$th relativistic image is defined as

$$
\mu_{n}=\frac{1}{\beta} \frac{\theta_{n}^{0} \varsigma_{n} D_{\mathrm{os}}}{D_{\mathrm{ls}}} .
$$

The first image is the outer most image having angular position $\theta_{1}$ and the remaining images are given as

$$
\theta_{\infty}=\frac{u_{\mathrm{ps}}}{D_{\mathrm{ol}}},
$$

where $\theta_{\infty}$ is the asymptotic position of the set of relativistic images obtained in the limit $n \rightarrow \infty$. For high alignment in the strong field limit, the observable (the angular separation $(S)$ between the first and remaining images and ratio $(r)$ between flux of the first and sum of other images) can be written as

$$
\begin{aligned}
& S=\theta_{\infty} e^{\left(a_{2}-2 \pi\right) / a_{1}}, \\
& r=e^{2 \pi / a_{1}} .
\end{aligned}
$$

Analysis of these parameters is useful to understand the nature of $\mathrm{BH}$ lens.

\section{Numerical Analysis of the Observables}

There is a strong evidence of the existence of supermassive BHs (with masses $10^{6}-10^{9}$ ) in the nuclei of galaxies of local universe [49-51]. It is believed that supermassive BHs at the galactic center have an intense effect on the evolution of host galaxies. Such BHs release a great amount of energy into surroundings which is helpful in star formation and growth of $\mathrm{BH}$ [52-54]. We examine the Einstein rings, magnifications, and observables in strong field by taking the example of supermassive $\mathrm{BH}$ in the center of galaxy NGC4486B, which has mass $5.7 \times 10^{8} M_{\odot}$ and distance from the Earth $D_{\mathrm{ol}}=$ 15.3 Mpc [55]. For the sake of simplicity, we assume that the lens is placed midway between the source and observer; that is, $D_{\mathrm{ls}} / D_{\mathrm{os}}=1 / 2[12]$.

The graphical analysis of observables is shown in Figure 6. For the case of ordinary global monopole, the increase in $\eta$ causes increase in the angular separation $S$ and angular 


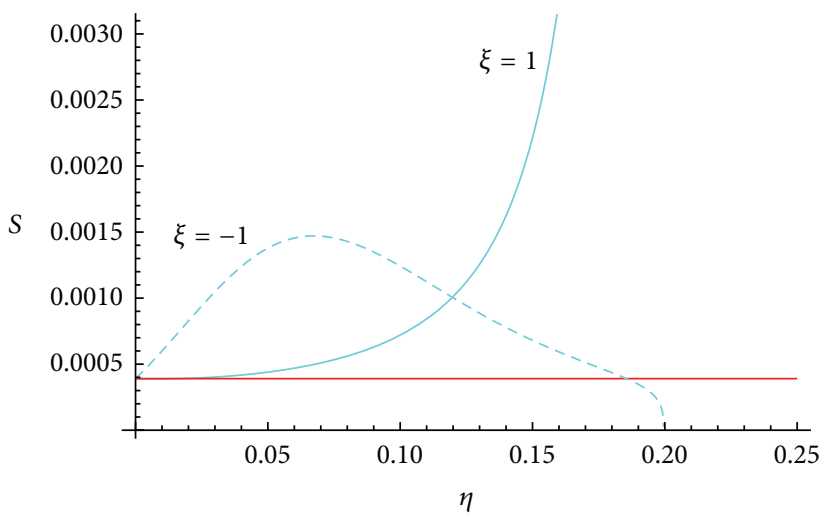

(a)

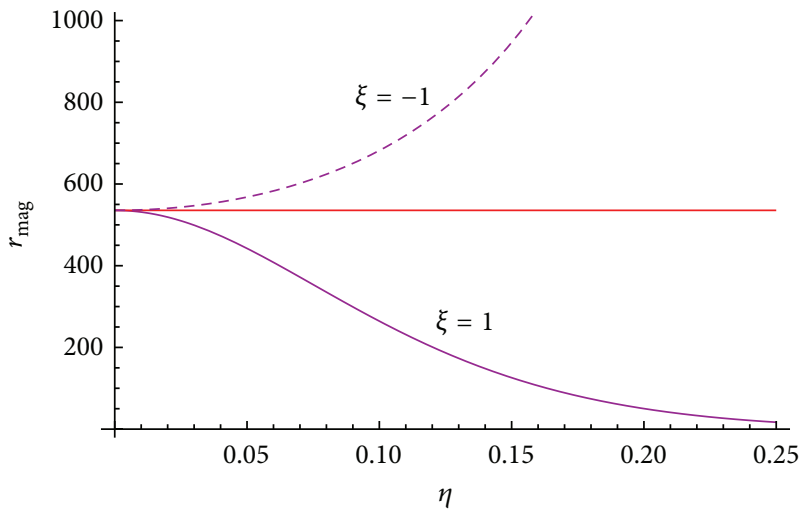

(b)

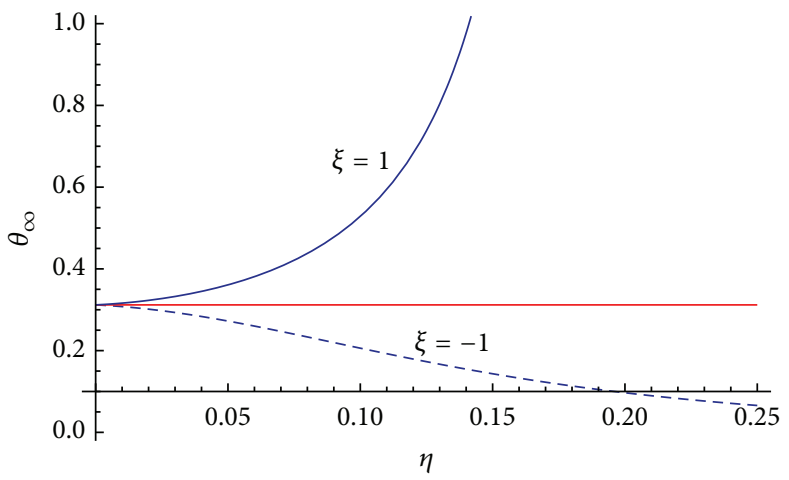

(c)

FIGURE 6: Plots of the observables corresponding to $\eta$. Red line shows the value for the Schwarzschild $\mathrm{BH}$.

TABLE 1: Einstein rings and magnifications $(n=1)$ for ordinary global monopole.

\begin{tabular}{lcccc}
\hline$\eta$ & 0.05 & 0.1 & 0.15 & 0.25 \\
$\mu_{n}$ & 0.1245 & 0.2510 & 4.6604 & 4.7497 \\
$\theta_{n}^{E}$ & 0.1872 & 0.2621 & 0.5825 & 0.7285 \\
\hline
\end{tabular}

TABLE 2: Einstein rings and magnifications $(n=2)$ for ordinary global monopole.

\begin{tabular}{ccccc}
\hline$\eta$ & 0.05 & 0.1 & 0.15 & 0.25 \\
$\mu_{n}$ & 0.0126 & 0.0170 & 0.3873 & 3.7923 \\
$\theta_{n}^{E}$ & 0.1866 & 0.2578 & 0.4263 & 0.6439 \\
\hline
\end{tabular}

position $\theta_{\infty}$; also both of these quantities are greater than those of Schwarzschild. The magnification of the outer most image $r_{\mathrm{mag}}=2.5 \log r$ appears as a decreasing function of $\eta$ and much less than Schwarzschild limit. For phantom global monopole, $S$ shows increasing behavior in the beginning but decreases gradually after $\eta \approx 0.075$. Thus, for low values of $\eta$, angular separation is greater while becoming smaller than Schwarzschild limit for high values of $\eta$ (after $\eta=0.19$ ). The magnification of the outer most image continuously increases and is greater than Schwarzschild limit while angular position is less than the Schwarzschild limit. We observe that for $\xi=1$ images become more separated for large values of $\eta$. However, for $\xi=-1$, images are well separated in the region
TABLE 3: Einstein rings and magnifications $(n=1)$ for phantom global monopole.

\begin{tabular}{lcccc}
\hline$\eta$ & 0.05 & 0.1 & 0.15 & 0.25 \\
$\mu_{n}$ & 0.0119 & 0.0691 & 0.0665 & 0.0301 \\
$\theta_{n}^{E}$ & 0.1550 & 0.1213 & 0.0867 & 0.0598 \\
\hline
\end{tabular}

TABle 4: Einstein rings and magnifications $(n=2)$ for phantom global monopole.

\begin{tabular}{lcccc}
\hline$\eta$ & 0.05 & 0.1 & 0.15 & 0.25 \\
$\mu_{n}$ & 0.03435 & 0.0172 & 0.0158 & 0.0102 \\
$\theta_{n}^{E}$ & 0.1522 & 0.1482 & 0.0830 & 0.0046 \\
\hline
\end{tabular}

$\eta=0.05-0.09$ and after $\eta=0.09$, it is difficult to distinguish between the first and the remaining images. The relative magnitude $r_{\text {mag }}$ for ordinary global monopole is smaller than the $\mathrm{BH}$ with phantom global monopole. The angular position $\theta_{\infty}$ for the case $\xi=1$ is larger than the case of $\xi=-1$.

Tables 1-4 indicate the numerical results for Einstein rings and image magnifications corresponding to the first $(n=1)$ and second images $(n=2)$. In the first case (ordinary global monopole), numbers of Einstein rings and magnifications gradually increase for both images $(n=1,2)$ but the image is brighter than the second one. Also, there exist more Einstein rings for the first image than the second. In the second case (phantom global monopole), the behavior 
of Einstein rings and magnifications is entirely different; both quantities decrease with increasing $\eta$; that is, strong lensing of BHs with phantom global monopole produces less Einstein rings and the images are fainter than the $\mathrm{BH}$ with global monopole.

\section{Time Delays between Relativistic Images}

In the strong field regime, the deflection angle (around a $\mathrm{BH}$ ) could be more than $2 \pi$ and multiple images might be formed. In this case, the time for the light propagation along each path corresponding to different images is not the same. Thus, intrinsic variations from a distant source may appear at different times in the multiple images. This time lag is called the time delay between two images of a lensed source. Refsdal [55] firstly proposed that Hubble parameter can be described by time delay. Following [56], we can calculate time delays between relativistic images.

The time delay between $m$ th and $n$th relativistic images from different paths of photons formed on the same side of the lens is given by [32]

$$
\begin{aligned}
& \Delta T_{n, m}^{s}=u_{\mathrm{ps}}[2 \pi(n-m) \\
& \quad+2 \sqrt{2}\left\{e^{\left(a_{2}-2 m \pi\right) / 2 a_{1}}-e^{\left(a_{2}-2 n \pi\right) / 2 a_{1}}\right\} \\
& \left. \pm \frac{\sqrt{2} D_{\mathrm{os}} \beta}{a_{1} D_{\mathrm{ls}}}\left(e^{\left(a_{2}-2 m \pi\right) / 2 a_{1}}-e^{\left(a_{2}-2 n \pi\right) / 2 a_{1}}\right)\right],
\end{aligned}
$$

where positive (negative) sign indicates if both images are on the same (opposite) side of the source. For images on the opposite side of the lens, we have [44]

$$
\begin{aligned}
& \Delta T_{n, m}^{o}=u_{\mathrm{ps}}[2 \pi(n-m) \\
& +2 \sqrt{2}\left\{e^{\left(a_{2}-2 m \pi\right) / 2 a_{1}}-e^{\left(a_{2}-2 n \pi\right) / 2 a_{1}}\right\} \\
& +\frac{\sqrt{2} D_{\mathrm{os}} \beta}{a_{1} D_{\mathrm{ls}}}\left(e^{\left(a_{2}-2 m \pi\right) / 2 a_{1}}-e^{\left(a_{2}-2 n \pi\right) / 2 a_{1}}\right) \\
& \left.-\frac{2 D_{\mathrm{os}} \beta}{D_{\mathrm{ls}}}\right] .
\end{aligned}
$$

The winding number $n$ denotes image on the same side of the source and $m$ represents image on the opposite side of the source. Some results for ordinary and phantom global monopoles are presented in Tables 5-10 for the specific masses and distances taken from [56].

In Tables 5-10, the subscripts + and - represent the case of both images which are on the same or opposite side of the source. We observe that, with increasing values of $\eta$, time delays for the $\mathrm{BH}$ with global monopole increase. The situation is totally opposite for the $\mathrm{BH}$ with phantom global monopole where the time delays have smaller values for larger values of $\eta$.
TABLE 5: Estimation of time delays of the supermassive BHs located at the center of galaxies for ordinary global monopole $(\xi=1)$ with $\eta=0.05$.

\begin{tabular}{lccccc}
\hline Galaxy & Mass $\left(M_{\odot}\right)$ & $\begin{array}{c}\text { Distance } \\
(\mathrm{Mpc})\end{array}$ & $\Delta T_{2,1}^{s+}(\mathrm{s})$ & $\Delta T_{2,1}^{s-}(\mathrm{s})$ & $\Delta T_{2,1}^{o}(\mathrm{~s})$ \\
\hline Milky way & $2.8 \times 10^{6}$ & 0.0085 & 23.7822 & 23.6985 & 17.1925 \\
NGC0224 & $3.0 \times 10^{7}$ & 0.7 & 27.8269 & 19.6380 & 7.8285 \\
NGC3115 & $2.0 \times 10^{9}$ & 8.4 & 72.8660 & 25.4010 & 10.4984 \\
NGC4594 & $1.0 \times 10^{9}$ & 9.0 & 76.3755 & 28.9100 & 38.0923 \\
NGC4486B & $5.7 \times 10^{8}$ & 15.3 & 79.1123 & 65.7607 & 51.0394 \\
NGC4261 & $4.5 \times 10^{8}$ & 27.4 & 113.2257 & 136.5364 & 75.9061 \\
NGC7052 & $3.3 \times 10^{8}$ & 58.7 & 184.0013 & 319.6172 & 140.2306 \\
NGC3377 & $1.8 \times 10^{8}$ & 9.9 & 81.6398 & 34.1749 & 39.9419 \\
\hline
\end{tabular}

TABLE 6: Estimation of time delays of the supermassive BHs located at the center of galaxies for ordinary global monopole $(\xi=1)$ with $\eta=0.2$.

\begin{tabular}{lccccc}
\hline Galaxy & Mass $\left(M_{\odot}\right)$ & $\begin{array}{c}\text { Distance } \\
(\mathrm{Mpc})\end{array}$ & $\Delta T_{2,1}^{s+}(\mathrm{s})$ & $\Delta T_{2,1}^{s-}(\mathrm{s})$ & $\Delta T_{2,1}^{o}(\mathrm{~s})$ \\
\hline Milky way & $2.8 \times 10^{6}$ & 0.0085 & 34.0185 & 23.3056 & 52.5924 \\
NGC0224 & $3.0 \times 10^{7}$ & 0.7 & 40.9103 & 32.4913 & 49.0863 \\
NGC3115 & $2.0 \times 10^{9}$ & 8.4 & $2.1 \times 10^{2}$ & 76.1015 & 89.4065 \\
NGC4594 & $1.0 \times 10^{9}$ & 9.0 & $1.3 \times 10^{2}$ & $1.7 \times 10^{2}$ & 119.3648 \\
NGC4486B & $5.7 \times 10^{8}$ & 15.3 & $3.3 \times 10^{2}$ & $1.3 \times 10^{3}$ & $1.8 \times 10^{2}$ \\
NGC4261 & $4.5 \times 10^{8}$ & 27.4 & $1.6 \times 10^{3}$ & $3.4 \times 10^{2}$ & $2.7 \times 10^{2}$ \\
NGC7052 & $3.3 \times 10^{8}$ & 58.7 & $3.4 \times 10^{2}$ & $2.2 \times 10^{2}$ & $2.3 \times 10^{2}$ \\
NGC3377 & $1.8 \times 10^{8}$ & 9.9 & $2.7 \times 10^{2}$ & $3.9 \times 10^{2}$ & $1.9 \times 10^{2}$ \\
\hline
\end{tabular}

TABLE 7: Estimation of time delays of the supermassive BHs located at the center of galaxies for ordinary global monopole $(\xi=1)$ with $\eta=0.25$.

\begin{tabular}{lccccc}
\hline Galaxy & Mass $\left(M_{\odot}\right)$ & $\begin{array}{c}\text { Distance } \\
(\mathrm{Mpc})\end{array}$ & $\Delta T_{2,1}^{s+}(\mathrm{s})$ & $\Delta T_{2,1}^{s-}(\mathrm{s})$ & $\Delta T_{2,1}^{o}(\mathrm{~s})$ \\
\hline Milky way & $2.8 \times 10^{6}$ & 0.0085 & $1.4 \times 10^{3}$ & $1.7 \times 10^{2}$ & $1.6 \times 10^{2}$ \\
NGC0224 & $3.0 \times 10^{7}$ & 0.7 & $1.7 \times 10^{4}$ & $2.4 \times 10^{3}$ & $1.6 \times 10^{4}$ \\
NGC3115 & $2.0 \times 10^{9}$ & 8.4 & $2.7 \times 10^{3}$ & $4.0 \times 10^{3}$ & $2.8 \times 10^{3}$ \\
NGC4594 & $1.0 \times 10^{9}$ & 9.0 & $1.9 \times 10^{2}$ & $5.5 \times 10^{2}$ & $2.0 \times 10^{3}$ \\
NGC4486B & $5.7 \times 10^{8}$ & 15.3 & $2.1 \times 10^{3}$ & $4.2 \times 10^{3}$ & $2.1 \times 10^{2}$ \\
NGC4261 & $4.5 \times 10^{8}$ & 27.4 & $3.2 \times 10^{3}$ & $4.3 \times 10^{3}$ & $3.2 \times 10^{2}$ \\
NGC7052 & $3.3 \times 10^{8}$ & 58.7 & $3.5 \times 10^{3}$ & $4.0 \times 10^{4}$ & $3.5 \times 10^{3}$ \\
NGC3377 & $1.8 \times 10^{8}$ & 9.9 & $3.3 \times 10^{2}$ & $5.2 \times 10^{3}$ & $2.3 \times 10^{2}$ \\
\hline
\end{tabular}


TABLE 8: Estimation of time delays of the supermassive BHs located at the center of galaxies for phantom global monopole $(\xi=-1)$ with $\eta=0.05$.

\begin{tabular}{lccccc}
\hline Galaxy & Mass $\left(M_{\odot}\right)$ & $\begin{array}{c}\text { Distance } \\
(\mathrm{Mpc})\end{array}$ & $\Delta T_{2,1}^{s+}(\mathrm{s})$ & $\Delta T_{2,1}^{s-}(\mathrm{s})$ & $\Delta T_{2,1}^{o}(\mathrm{~s})$ \\
\hline Milky way & $2.8 \times 10^{6}$ & 0.0085 & $1.7 \times 10^{7}$ & $1.5 \times 10^{5}$ & $4.9 \times 10^{4}$ \\
NGC0224 & $3.0 \times 10^{7}$ & 0.7 & $3.4 \times 10^{5}$ & $7.7 \times 10^{5}$ & $3.2 \times 10^{3}$ \\
NGC3115 & $2.0 \times 10^{9}$ & 8.4 & $2.2 \times 10^{4}$ & $1.9 \times 10^{5}$ & $1.7 \times 10^{4}$ \\
NGC4594 & $1.0 \times 10^{9}$ & 9.0 & $2.4 \times 10^{4}$ & $2.0 \times 10^{4}$ & $1.3 \times 10^{4}$ \\
NGC4486B & $5.7 \times 10^{8}$ & 15.3 & $4.0 \times 10^{4}$ & $3.6 \times 10^{4}$ & $6.3 \times 10^{3}$ \\
NGC4261 & $4.5 \times 10^{8}$ & 27.4 & $7.0 \times 10^{5}$ & $9.7 \times 10^{6}$ & $3.3 \times 10^{5}$ \\
NGC7052 & $3.3 \times 10^{8}$ & 58.7 & $1.4 \times 10^{5}$ & $8.5 \times 10^{4}$ & $1.4 \times 10^{4}$ \\
NGC3377 & $1.8 \times 10^{8}$ & 9.9 & $2.6 \times 10^{6}$ & $2.3 \times 10^{5}$ & $1.9 \times 10^{3}$ \\
\hline
\end{tabular}

TABLE 9: Estimation of time delays of the supermassive BHs located at the center of galaxies for phantom global monopole $(\xi=-1)$ with $\eta=0.2$.

\begin{tabular}{lccccc}
\hline Galaxy & Mass $\left(M_{\odot}\right)$ & $\begin{array}{c}\text { Distance } \\
(\mathrm{Mpc})\end{array}$ & $\Delta T_{2,1}^{s+}(\mathrm{s})$ & $\Delta T_{2,1}^{s-}(\mathrm{s})$ & $\Delta T_{2,1}^{o}(\mathrm{~s})$ \\
\hline Milky way & $2.8 \times 10^{6}$ & 0.0085 & $1.2 \times 10^{2}$ & $1.5 \times 10^{2}$ & $1.3 \times 10^{2}$ \\
NGC0224 & $3.0 \times 10^{7}$ & 0.7 & $4.1 \times 10^{3}$ & $7.3 \times 10^{2}$ & $2.7 \times 10^{2}$ \\
NGC3115 & $2.0 \times 10^{9}$ & 8.4 & $5.2 \times 10^{3}$ & $1.8 \times 10^{4}$ & $2.3 \times 10^{2}$ \\
NGC4594 & $1.0 \times 10^{9}$ & 9.0 & $3.4 \times 10^{3}$ & $2.7 \times 10^{3}$ & $4.3 \times 10^{3}$ \\
NGC4486B & $5.7 \times 10^{8}$ & 15.3 & $3.7 \times 10^{4}$ & $2.2 \times 10^{3}$ & $3.2 \times 10^{3}$ \\
NGC4261 & $4.5 \times 10^{8}$ & 27.4 & $6.1 \times 10^{5}$ & $9.3 \times 10^{6}$ & $4.7 \times 10^{2}$ \\
NGC7052 & $3.3 \times 10^{8}$ & 58.7 & $9.2 \times 10^{3}$ & $3.5 \times 10^{3}$ & $1.8 \times 10^{2}$ \\
NGC3377 & $1.8 \times 10^{8}$ & 9.9 & $2.8 \times 10^{4}$ & $7.3 \times 10^{4}$ & $1.2 \times 10^{3}$ \\
\hline
\end{tabular}

TABLE 10: Estimation of time delays of the supermassive BHs located at the center of galaxies for phantom global monopole $(\xi=-1)$ with $\eta=0.25$.

\begin{tabular}{lccccc}
\hline Galaxy & Mass $\left(M_{\odot}\right)$ & $\begin{array}{c}\text { Distance } \\
(\mathrm{Mpc})\end{array}$ & $\Delta T_{2,1}^{s+}(\mathrm{s})$ & $\Delta T_{2,1}^{s-}(\mathrm{s})$ & $\Delta T_{2,1}^{o}(\mathrm{~s})$ \\
\hline Milky way & $2.8 \times 10^{6}$ & 0.0085 & 3.9 & 3.0 & 0.095 \\
NGC0224 & $3.0 \times 10^{7}$ & 0.7 & $3.3 \times 10^{3}$ & $3.4 \times 10^{2}$ & $2.8 \times 10^{-2}$ \\
NGC3115 & $2.0 \times 10^{9}$ & 8.4 & $3.6 \times 10^{3}$ & $3.2 \times 10^{3}$ & $3.9 \times 10^{-4}$ \\
NGC4594 & $1.0 \times 10^{9}$ & 9.0 & $4.1 \times 10^{2}$ & $4.3 \times 10^{2}$ & $5.3 \times 10^{-3}$ \\
NGC4486B & $5.7 \times 10^{8}$ & 15.3 & $8.3 \times 10^{3}$ & $8.4 \times 10^{2}$ & $3.8 \times 10^{-2}$ \\
NGC4261 & $4.5 \times 10^{8}$ & 27.4 & $9.7 \times 10^{4}$ & $8.9 \times 10^{6}$ & $8.2 \times 10^{-2}$ \\
NGC7052 & $3.3 \times 10^{8}$ & 58.7 & $8.5 \times 10^{3}$ & $8.6 \times 10^{2}$ & $8.3 \times 10^{-3}$ \\
NGC3377 & $1.8 \times 10^{8}$ & 9.9 & $8.4 \times 10^{4}$ & $8.7 \times 10^{3}$ & $8.2 \times 10^{-4}$ \\
\hline
\end{tabular}

\section{Final Remarks}

In this paper, we have examined the effects of energy scale of symmetry breaking $\eta$ on the null geodesics as well as strong gravitational lensing for $\mathrm{BHs}$ having ordinary and phantom global monopoles. The results are shown graphically and are compared with the Schwarzschild $\mathrm{BH}$. The analysis of $V_{\text {eff }}$ represents that only unstable orbits of particles exist for $\xi=$ -1 , whereas $V_{\text {eff }}$ for $\xi=1$ leads to stable circular orbits. We have adopted the technique of Bozza to calculate the deflection angle and all the related quantities for both cases $(\xi=1,-1)$. The deflection angle for the $\mathrm{BH}$ with ordinary global monopole is monotonically increasing function of $\eta$ and larger than the Schwarzschild limit. For the phantom global monopole, the deflection angle continuously decreases and is less than the deflection angle of Schwarzschild as well as $\mathrm{BH}$ with ordinary global monopole. The behavior of deflection angle for phantom global monopole is entirely different from [35], where $\alpha(\theta)$ is an increasing function of phantom constant $b$.

We have considered the example of supermassive $\mathrm{BH}$ at the center of galaxy NGC4486B for Einstein rings, magnifications, and observables. For the $\mathrm{BH}$ with ordinary global monopole, the images are well separated for high values of $\eta$. The inner most angular positions are greater while relative magnitude is smaller than the Schwarzschild limit. The analysis of phantom global monopole shows that the separation among images is high for initial values of $\eta$ but it gradually reduces when $\eta$ increases. The inner most angular positions are less while relative magnitude is larger than Schwarzschild BH. When $\xi=1$, images are more prominent and formation of Einstein rings increases as $\eta$ increases. For the $\mathrm{BH}$ with phantom global monopole $(\xi=-1)$, the images become fade for large $\eta$, and also numbers of Einstein rings gradually decrease. It is worth mentioning that the results of observables for the case of phantom global monopole are contrary to [35].

Finally, we have studied the time delays between relativistic images by taking the example of different galaxies having supermassive $\mathrm{BH}$ in their center. We have considered two cases for both ordinary and global monopoles, when the images (first and second) are on the same side of the lens and when the images are on the opposite side of the lens. The time delays for an ordinary global monopole become larger with large values of $\eta$, whereas for phantom global monopole they have smaller values as $\eta$ increases. We conclude that the parameter $\eta$ (energy scale of symmetry breaking) highly affects the propagation of light in the strong field limit. It is worthwhile to mention here that the strong field lensing properties in both cases (ordinary and phantom global monopoles) are different not only from each other but also from the Schwarzschild $\mathrm{BH}$.

\section{Appendix}

The components of energy-momentum tensor for metric (1) can be written as

$$
\begin{aligned}
& T_{0}^{0}=T_{1}^{1}=-\frac{f^{\prime}}{r}-\frac{f}{r^{2}}+\frac{1}{r^{2}}, \\
& T_{2}^{2}=T_{3}^{3}=-\frac{f^{\prime}}{r}-\frac{f^{\prime \prime}}{2} .
\end{aligned}
$$


The appropriate general expressions of the spherically symmetric spacetime for phantom global monopole are [57]

$$
\begin{aligned}
& T_{0}^{0}=\rho_{p}(r), \\
& T_{i}^{j}=3 \rho_{p}(r) \omega_{p}\left[-(1+3 B) \frac{r_{i} r^{j}}{r^{n} r_{n}}+B \delta_{i}^{j}\right] .
\end{aligned}
$$

This leads the spatial part of the energy-momentum tensor proportional to the time component with the arbitrary parameter $B$ which depends on the internal structure of phantom fields. The isotropic averaging over the angle results in

$$
\left\langle T_{i}^{j}\right\rangle=-\rho_{p}(r) \omega_{p} \delta_{i}^{j}=-P_{p}(r) \delta_{i}^{j},
$$

where $P_{p}=\omega_{p} \rho_{p}(r)$. Using the above equations, the energymomentum has the form

$$
\begin{aligned}
& T_{0}^{0}=T_{1}^{1}=\rho_{p}, \\
& T_{2}^{2}=T_{3}^{3}=-\frac{1}{2}\left(3 \omega_{p}+1\right) \rho_{p} .
\end{aligned}
$$

Using (A.1) and (A.4), we write the expression for pressure as

$$
P_{p}(r)=\frac{f^{\prime}}{r}+\frac{f^{\prime \prime}}{3}+\frac{f}{3 r^{2}}-\frac{1}{3 r^{2}} .
$$

\section{Conflict of Interests}

The authors declare that there is no conflict of interests regarding the publication of this paper.

\section{References}

[1] S. Chandrasekhar, The Mathematical Theory of Black Holes, Oxford University Press, 1983.

[2] S. Fernando, D. Krug, and C. Curry, "Geodesic structure of static charged black hole solutions in $2+1$ dimensions," General Relativity and Gravitation, vol. 35, no. 7, pp. 1243-1261, 2003.

[3] R. A. Konoplya, "Particle motion around magnetized black holes: preston-poisson space-time," Physical Review D, vol. 74, no. 12, Article ID 124015, 2006.

[4] C. Leiva, J. Saavedra, and J. Villanueva, "Geodesic structure of the Schwarzschild black hole in rainbow gravity," Modern Physics Letters A: Particles and Fields, Gravitation, Cosmology, Nuclear Physics, vol. 24, no. 18, article 1443, 2009.

[5] S. Guha and P. Bhattacharya, "Geodesic motions near a fivedimensional reissner-nordström anti-de sitter black hole," Journal of Physics: Conference Series, vol. 405, Article ID 012017, 2012.

[6] P. Pradhan, "Circular geodesics in the Kerr-Newman-TaubNUT spacetime," Classical and Quantum Gravity, vol. 32, no. 16, Article ID 165001, 2015.

[7] C. Darwin, "The gravity field of a particle," Proceedings of the Royal Society of London A, vol. 249, p. 180, 1959.

[8] D. Walsh, R. F. Carswell, and R. J. Weymann, “0957+561 A, B: twin quasistellar objects or gravitational lens?” Nature, vol. 279, no. 5712, pp. 381-384, 1979.

[9] J. N. Hewitt, E. L. Turner, C. R. Lawrence, D. P. Schneider, and J. P. Brody, "A gravitational lens candidate with an unusually red optical counterpart," Astronomical Journal, vol. 104, no. 3, pp. 968-979, 1992.
[10] S. U. Viergutz, "Image generation in Kerr geometry. I. Analytical investigations on the stationary emitter-observer problem," Astronomy and Astrophysics, vol. 272, no. 1, pp. 355-377, 1993.

[11] H. Falcke, F. Melia, and E. Agol, "Viewing the shadow of the black hole at the galactic center," The Astrophysical Journal, vol. 528, no. 1, pp. L13-L16, 2000.

[12] K. S. Virbhadra and G. F. R. Ellis, "Schwarzschild black hole lensing," Physical Review D, vol. 62, no. 8, Article ID 084003, 2000.

[13] S. Frittelli, T. P. Kling, and E. T. Newman, "Spacetime perspective of Schwarzschild lensing," Physical Review D, vol. 61, no. 6, Article ID 064021, 14 pages, 2000.

[14] V. Bozza, "Gravitational lensing in the strong field limit," Physical Review D, vol. 66, Article ID 103001, 2002.

[15] A. Y. Bin-Nun, "Gravitational lensing of stars orbiting Sgr A* as a probe of the black hole metric in the Galactic center," Physical Review D, vol. 82, Article ID 064009, 2010.

[16] A. Y. Bin-Nun, "Strong gravitational lensing by Sgr A*," Classical and Quantum Gravity, vol. 28, no. 11, Article ID 114003, 2011.

[17] C. Ding, S. Kang, and C.-Y. Chen, "Strong gravitational lensing in a noncommutative black-hole spacetime," Physical Review D, vol. 83, Article ID 084005, 2011.

[18] J. Deng, "Strong field gravitational lensing in a magnetic charged Reissner-Nordström black hole pierced by a cosmic string," International Journal of Theoretical Physics, vol. 51, no. 5, pp. 1632-1639, 2012.

[19] S. Sahu, M. Patil, D. Narasimha, and P. S. Joshi, "Can strong gravitational lensing distinguish naked singularities from black holes?” Physical Review D, vol. 86, Article ID 063010, 2012.

[20] S.-W. Wei, Y.-X. Liu, C.-E. Fu, and K. Yang, "Strong field limit analysis of gravitational lensing in Kerr-Taub-NUT spacetime," Journal of Cosmology and Astroparticle Physics, vol. 2012, no. 10, article 053, 2012.

[21] N. Bilić, H. Nikolić, and R. D. Viollier, "Fermion stars as gravitational lenses," The Astrophysical Journal, vol. 537, no. 2, pp. 909-915, 2000.

[22] K. K. Nandi, Y. Z. Zhang, and A. V. Zakharov, "Gravitational lensing by wormholes," Physical Review D, vol. 74, no. 2, Article ID 024020, 13 pages, 2006.

[23] G. Gyulchev and S. Yazadjiev, "Gravitational lensing by rotating naked singularities in the equatorial plane," AIP Conference Proceedings, vol. 946, no. 1, pp. 106-118, 2007.

[24] R. A. Konoplya, "Magnetised black hole as a gravitational lens," Physics Letters B, vol. 644, no. 4, pp. 219-223, 2007.

[25] A. M. Nzioki, P. K. S. Dunsby, R. Goswami, and S. Carloni, "Geometrical approach to strong gravitational lensing in $f(R)$ gravity," Physical Review D, vol. 83, no. 2, Article ID 024030, 10 pages, 2011.

[26] T. Kitamura, K. Nakajima, and H. Asada, "Demagnifying gravitational lenses toward hunting a clue of exotic matter and energy," Physical Review D, vol. 87, no. 2, Article ID 027501, 5 pages, 2013.

[27] K. Izumi, C. Hagiwara, K. Nakajima, T. Kitamura, and H. Asada, "Gravitational lensing shear by an exotic lens object with negative convergence or negative mass," Physical Review D, vol. 88, Article ID 024049, 2013.

[28] M. Sharif and S. Iftikhar, "Charged dissipative collapse of shearing viscous star," Astrophysics and Space Science, vol. 357, article 79, 2015. 
[29] M. Sharif and S. Iftikhar, "Null geodesics and strong field gravitational lensing in a string cloud background," Advances in High Energy Physics, vol. 2015, Article ID 635625, 9 pages, 2015.

[30] E. Babichev, V. Dokuchaev, and Y. Eroshenko, "Black hole mass decreasing due to phantom energy accretion," Physical Review Letters, vol. 93, no. 2, Article ID 021102, 4 pages, 2004.

[31] K. A. Bronnikov and J. C. Fabris, "Regular phantom black holes," Physical Review Letters, vol. 96, no. 25, Article ID 251101, 2006.

[32] E. F. Eiroa and C. M. Sendra, "Regular phantom black hole gravitational lensing," Physical Review D, vol. 88, Article ID 103007, 2013.

[33] M. Azreg-Aïnou, "Light paths of normal and phantom EinsteinMaxwell-dilaton black holes," Physical Review D, vol. 87, no. 2, Article ID 024012, 20 pages, 2013.

[34] G. N. Gyulchev and I. Z. Stefanov, "Gravitational lensing by phantom black holes," Physical Review D, vol. 87, no. 6, Article ID 063005, 12 pages, 2013.

[35] C. Ding, C. Liu, Y. Xiao, L. Jiang, and R.-G. Cai, "Strong gravitational lensing in a black-hole spacetime dominated by dark energy," Physical Review D, vol. 88, Article ID 104007, 2013.

[36] M. Barriola and A. Vilenkin, "Gravitational field of a global monopole," Physical Review Letters, vol. 63, no. 4, pp. 341-343, 1989.

[37] F. D. Mazzitelli and C. O. Lousto, "Vacuum-polarization effects in global monopole space-times," Physical Review D, vol. 43, no. 2, article 468, 1991.

[38] H. Koyama and A. Tomimatsu, "Asymptotic tails of massive scalar fields in a Schwarzschild background," Physical Review D, vol. 64, no. 4, Article ID 044014, 2001.

[39] K. A. Bronnikov, B. E. Meierovich, and E. R. Podolyak, "Global monopole in general relativity," Journal of Experimental and Theoretical Physics, vol. 95, no. 3, pp. 392-403, 2002.

[40] H. Yu, "Decay of massive scalar hair in the background of a black hole with a global monopole," Physical Review D, vol. 65, Article ID 087502, 2002.

[41] J. P. M. Pitelli and P. S. Letelier, "Quantum singularities around a global monopole," Physical Review D, vol. 80, Article ID 104035, 2009.

[42] F. Rahaman and B. C. Bhui, "Gravitational field of a non-stationary global monopole in higher dimension," Fizika B, vol. 14, no. 4, pp. 349-356, 2005.

[43] S. Chen and J. Jing, "Gravitational field of a slowly rotating black hole with a phantom global monopole," Classical and Quantum Gravity, vol. 30, no. 17, Article ID 175012, 2013.

[44] C.-M. Claudel, K. S. Virbhadra, and G. F. R. Ellis, “The geometry of photon surfaces," Journal of Mathematical Physics, vol. 42, no. 2, pp. 818-838, 2001.

[45] S. Weinberg, Gravitation and Cosmology: Principles and Applications of the General Theory of Relativity, Wiley, 1972.

[46] K. S. Virbhadra, D. Narasimha, and S. M. Chitre, "Role of the scalar field in gravitational lensing," Astronomy and Astrophysics, vol. 337, no. 1, pp. 1-8, 1998.

[47] K. S. Virbhadra and G. F. Ellis, "Gravitational lensing by naked singularities," Physical Review D, vol. 65, no. 10, Article ID 103004, 10 pages, 2002.

[48] V. Bozza, S. Capozziello, G. Iovane, and G. Scarpetta, "Strong field limit of black hole gravitational lensing," General Relativity and Gravitation, vol. 33, no. 9, pp. 1535-1548, 2001.
[49] J. Kormendy and D. Richstone, "Inward bound-the search for supermassive black holes in galactic nuclei," Annual Review of Astronomy and Astrophysics, vol. 33, no. 1, pp. 581-624, 1995.

[50] J. Magorrian, S. Tremaine, D. Richstone et al., "The demography of massive dark objects in galaxy centers," The Astronomical Journal, vol. 115, no. 6, pp. 2285-2305, 1998.

[51] L. Ferrarese and H. Ford, "Supermassive black holes in galactic nuclei: past, present and future research," Space Science Reviews, vol. 116, no. 3-4, pp. 523-624, 2005.

[52] J. Silk and M. J. Rees, "Quasars and galaxy formation," Astronomy \& Astrophysics, vol. 331, pp. L1-L4, 1998.

[53] A. King, "Black holes, galaxy formation, and the $M_{\mathrm{BH}^{-}}-$ relation," Monthly Notices of the Royal Astronomical Society, vol. 596, no. 1, pp. L27-L29, 2003.

[54] J. S. B. Wyithe and A. Loeb, "Self-regulated growth of supermassive black holes in galaxies as the origin of the optical and X-ray luminosity functions of quasars," The Astrophysical Journal, vol. 595, no. 2, article 614, 2003.

[55] S. Refsdal, "On the possibility of determining Hubble's parameter and the masses of galaxies from the gravitational lens effect," Monthly Notices of the Royal Astronomical Society, vol. 128, pp. 307-310, 1964.

[56] V. Bozza and L. Mancini, “Time delay in black hole gravitational lensing as a distance estimator," General Relativity and Gravitation, vol. 36, no. 2, pp. 435-450, 2004.

[57] V. V. Kiselev, "Quintessence and black holes," Classical and Quantum Gravity, vol. 20, no. 6, article 1187, 2003. 

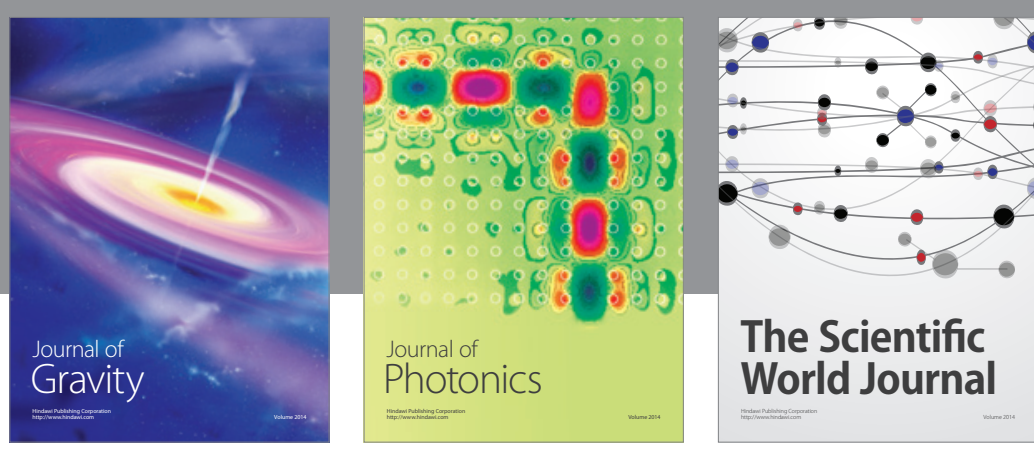

The Scientific World Journal
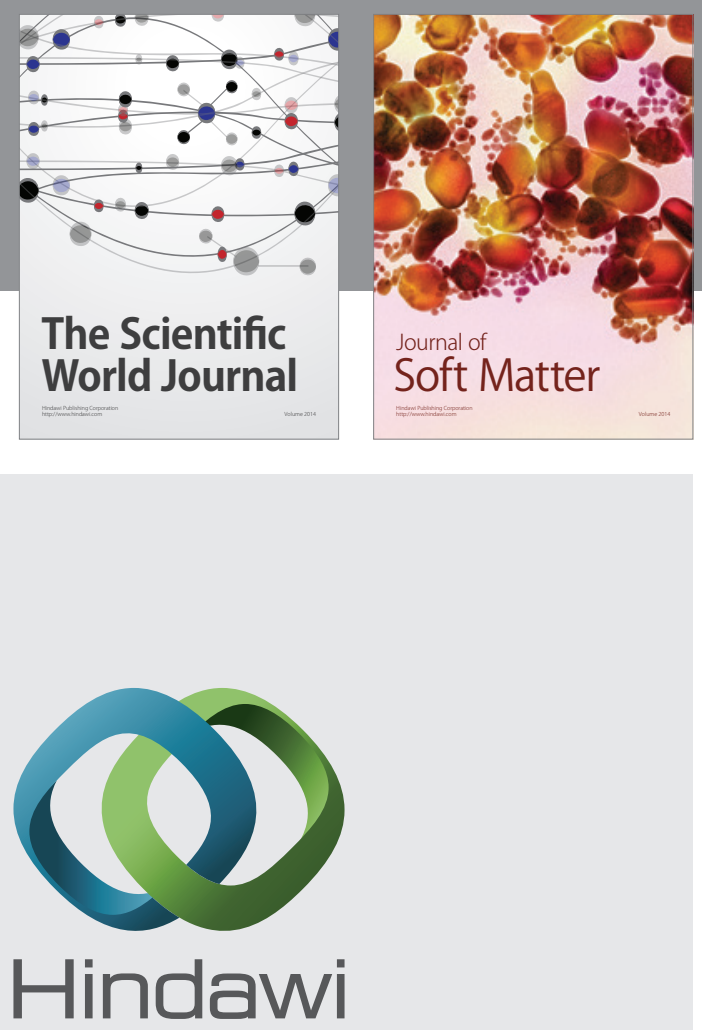

Submit your manuscripts at

http://www.hindawi.com

nternational Journal of

Statistical Mechanics
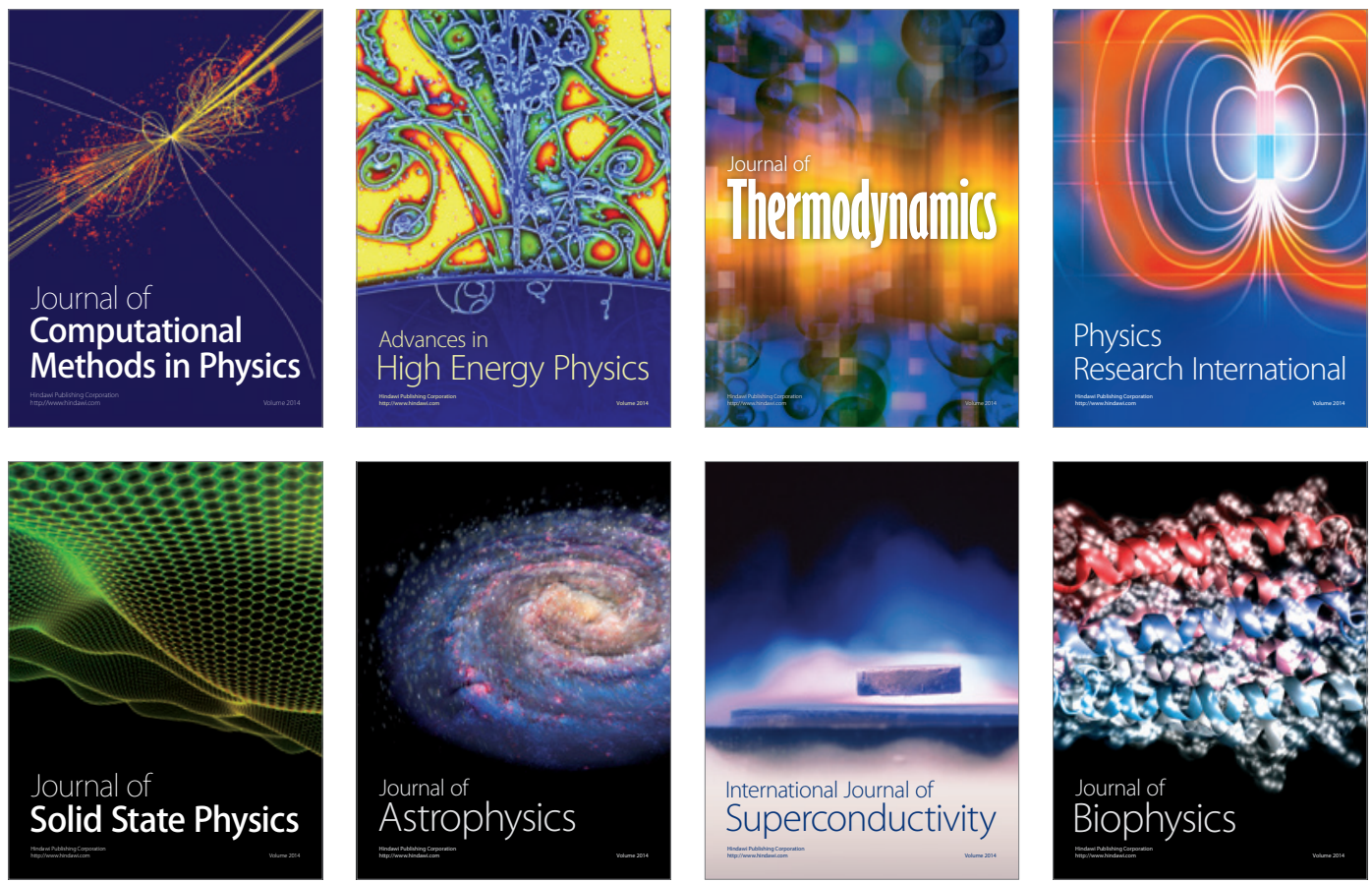
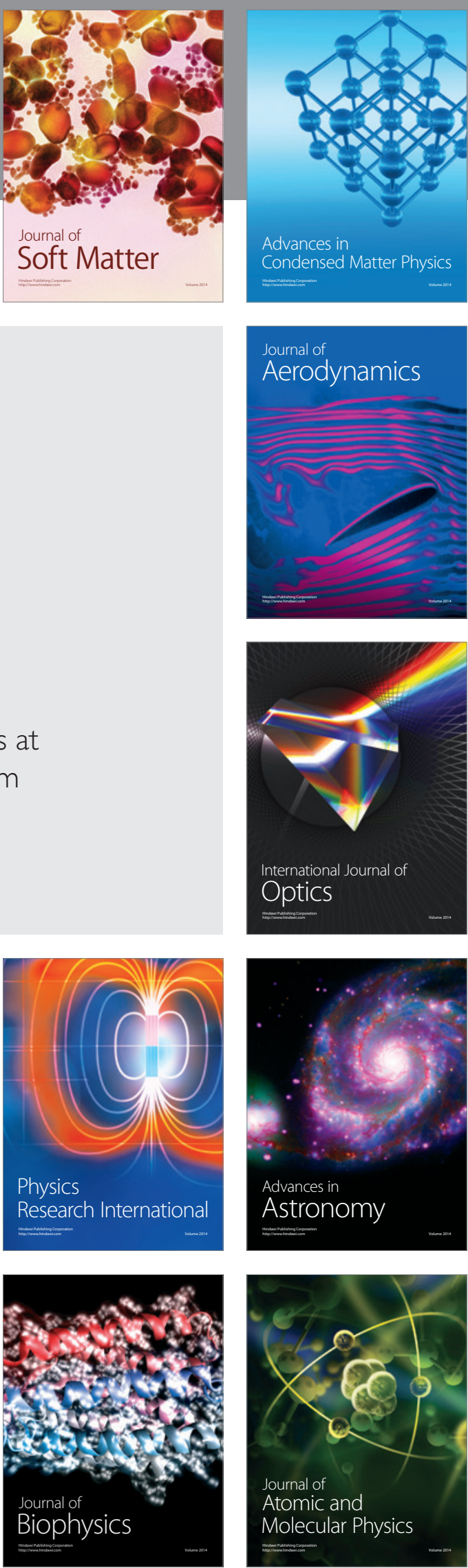